\title{
EDAD DE IMPUTABILIDAD PENAL Y JURISDICCIÓN DE LOS TRIBUNALES JUVENILES EN EUROPA*
}

\section{Imputability Age of Criminal and Jurisdiction of Juvenile Courts in Europe}

Frieder Dünkel l* $^{*}$

Resumen: El presente artículo realiza un análisis comparado de las principales características de los sistemas de justicia penal juvenil en Europa. En concreto se comparan las edades de imputabilidad penal, la problemática de los menores adultos, los tribunales juveniles y el rol que juegan los participantes en el proceso penal juvenil: defensor, juez, fiscal, víctima, imputado e instituciones sociales.

Palabras clave: edad de imputabilidad criminal - derecho penal juvenil - derecho procesal penal juvenil - política criminal

\begin{abstract}
The aim of this paper consists of a compared study of the main characteristics of the juvenile penal systems in Europe. This comparative study showsthe ages of penal liabibility in different legislations, the problems related to minor adult crimes, the juvenile courts and the roll that participants play in the juvenile penal process.
\end{abstract}

Keywords: age for criminal liability - juvenile criminal justice - juvenile criminal procedure in Europe - criminal policy

\section{1. Ámbito de aplicación del derecho penal y procesal penal juvenil}

\subsection{Limites de edad en la responsabilidad penal: imputabilidad}

Los límites de edad de responsabilidad penal son muy diferentes en los diversos países de Europa al igual como las normas especiales que regulan la aplicación de las sanciones penales juveniles. Incluso las recomendaciones contenidas en los estándares internacionales en este ámbito son diversas, por ejemplo, las Reglas de Beijing de las Naciones Unidas (1985), la Convención de los Derechos del niño

\footnotetext{
*El presente artículo se basa en los resultados obtenidos en el estudio sobre Justicia Penal Juvenil en Europa, el que fue elaborado en el marco del Programa de Justicia Criminal de la Unión Europea y gracias el financiamiento del Ministerio de Educación del Bundes Lander MecklenburgVorpommern. El estudio fue realizado por el Centro de Criminología de la Universidad de Greifswald entre los años 2006 y 2011 y contó con el trabajo conjunto de 34 universidades europeas, véase DÜNKEL / GRZYWA / HORSFIELD / PRUIN (2011); además DÜNKEL (2008).

** Profesor y director del Centro de Derecho Penal y Criminología de la Universidad de ErnstMoritz-Arndt-Greifswald de Alemania, correo electrónico: duenkel@uni-greifswald.de. Traducción del Dr. Álvaro Castro Morales.
} 
Dünkel: Edad de imputabilidad penal y jurisdicción de los tribunales juveniles en Europa

(CDN) de $1989^{1}$ o las Reglas Europeas para infractores menores de edad sometidos a sanciones y medidas (ERJOSSM) de 2008.

Las reglas europeas (ERJOSSM) se ocupan de los límites de edad de la responsabilidad penal juvenil y establecen lo que sigue: Para la imposición de una sanción o medida como reacción de un delito, es necesario que la edad minima no deba ser muy baja y asimismo legalmente determinada y medida.

Al igual que las Reglas de Beijing y las anteriores reglas europeas, las recomendaciones actuales (ERJOSSM) contienen, en contraste con la heterogeneidad de los límites de edad vigentes en Europa, una clara voluntad (cf. la tabla 1). El estándar de una edad no muy baja no es reconocido en países como Inglaterra y Gales, Irlanda o Suiza, los que contemplan la edad de 10 años, alejándose del promedio europeo de los 14 años. Para estos casos existen exigencias como las contenidas en el comentario de las reglas europeas, donde se contempla que los países que tienen una baja edad de imputabilidad debieran exigir una edad mayor para poder aplicar sanciones privativas de libertad. ${ }^{2}$ Llama la atención el caso de Suiza donde la sanción privativa de libertad solo puede ser impuesta desde los 15 años. ${ }^{3}$

En 18 de los 35 países analizados se establece la imputabilidad penal en la edad de 14 años. En los cuatro países escandinavos, más Grecia y República Checa en 15 años; en Portugal, 16 años; ${ }^{4}$ y en Bélgica, que sigue un modelo tutelar, para los delitos de tráfico de estupefacientes y delitos de especial gravedad (delitos violentos) desde 16 años. Solo nueve países establecen la imputabilidad penal bajo los 14 años. La imputabilidad penal desde 13 años existe en Francia y Polonia, ${ }^{5}$ en

${ }^{1}$ El Art. 40 3a) de la Convención de los Derechos del Niño exige de forma bastante suave que los Estados Partes están obligados a establecer una edad mínima, sin hacer una propuesta concreta en torno a ella. Sin embargo, el Comité de los Derechos del Niño de las Naciones Unidas publicó en 2007 el "General Comment No. 10 on Children's Rights in Juvenile Justice”, en el que recomienda como máximo la edad mínima de 12 años e incentiva a los Estados Partes a considerar una edad de imputablidad superior. Véase DOEK (2009), p. 23.

${ }^{2}$ COUnCIl OF Europe (2009), p. 36; DÜNKEL (2011), pp. 142 y s.

3 Para un análisis comparado véase PRUIN (2011), pp. 1539-1582; DÜNKEL / GRZYWA / PRUIN / ŠELIH (2011), pp. 1839-1898. En Escocia se modificó en 2010 la edad de imputabilidad de ocho a doce (Criminal Justice and Licensing [Scotland] Act 2010). De todas formas un paso correcto en dirección de los aceptados estándares europeos, los que proponen la edad mínima de 14 o 15 años. Véase BOCHMANN (2009), p. 88 y ss., quien es partidario de establecer un estándar europeo de 14 años.

${ }^{4} \mathrm{La}$ edad de los 12 años marca el umbral de intervención de los jueces juveniles bajo el modelo tutelar de países como Polonia. Véase Rodrigues / DuARTE-FOnSECA (2011), pp. 1027 y ss.

${ }^{5}$ En Polonia se considera la edad de 13 años, no en un sentido de imputabilidad penal, sino en el marco de competencia de los tribunales de familia. La edad general para aplicar sanciones penales es desde los 17, sin embargo, en el caso de delitos violentos pueden aplicarse sanciones penales desde los 15 años. Véase STAŃDO-KAWECKA (2011), pp. 991 y ss. 
12 años en Irlanda, ${ }^{6}$ Holanda, Escocia ${ }^{7}$ y Turquía. Finalmente tres países (Inglaterra y Gales, Irlanda del Norte y Suiza) fijan la responsabilidad penal desde los 10 años, con la salvedad de Suiza, donde -como ya mencionamos- la pena privativa de libertad solo puede ser aplicada desde los 15 años.

Tabla 1: Edades de imputabilidad penal y edades en que conocen los tribunales juveniles en Europa: Comparación

\begin{tabular}{|c|c|c|c|}
\hline País & $\begin{array}{l}\text { Edad } \\
\text { imputabilidad }\end{array}$ & $\begin{array}{l}\text { Edad en que el } \\
\text { derecho penal adulto } \\
\text { puede/debe aplicarse }\end{array}$ & $\begin{array}{l}\text { Edades en que conocen los } \\
\text { tribunales juveniles }\end{array}$ \\
\hline Bélgica & 16 ***/****/18 & $16 / 18$ & 14-18 \\
\hline Bulgaria & 14 & 18 & $14-18$ \\
\hline Dinamarca* & 15 & $15 / 18 / 21$ & $\begin{array}{l}\text { No hay jurisdicción penal } \\
\text { juvenil }\end{array}$ \\
\hline Alemania & 14 & $18 / 21$ & $14-21$ \\
\hline Inglaterra/Gales & $10 / 12 / 15^{* *}$ & 18 & $10-18$ \\
\hline Estonia & 14 & 18 & Tribunales penales generales \\
\hline Finlandia* & 15 & $15 / 18$ & $\begin{array}{l}\text { No hay jurisdicción penal } \\
\text { juvenil }\end{array}$ \\
\hline Francia & 13 & 18 & $13-18$ \\
\hline Grecia & 15 & $18 / 21$ & $15-18$ \\
\hline Irlanda & $12 / 16^{* *}$ & 18 & $12-18$ \\
\hline Italia & 14 & $18 / 21$ & $14-18$ \\
\hline Croacia & $14 / 16^{* *}$ & $18 / 21$ & $14-21$ \\
\hline Letonia & 14 & 18 & Tribunales penales generales \\
\hline Lituania & $14 * * * * / 16$ & $18 / 21$ & Tribunales penales generales \\
\hline Montenegro & $14 / 16^{* *}$ & $18 / 21$ & $14-21$ \\
\hline Holanda & 12 & $16 / 18 / 23$ & $12-18$ \\
\hline Irlanda del Norte & 10 & $17 / 18 / 21$ & $10-18$ \\
\hline Noruega* & 15 & 18 & $\begin{array}{l}\text { No hay jurisdicción penal } \\
\text { juvenil }\end{array}$ \\
\hline Austria & 14 & $18 / 21$ & $14-21$ \\
\hline Polonia & $13 * * * * *$ & $15 / 17 / 18$ & $13-18$ \\
\hline Portugal & $12 * * * * * / 16$ & $16 / 21$ & $12-16$ \\
\hline Rumania & $14 / 16$ & $18 /(20)$ & Tribunales penales generales \\
\hline Rusia & $14 * * * * / 16$ & $18 / 21$ & Tribunales penales generales \\
\hline Suecia* & 15 & $15 / 18 / 21$ & $\begin{array}{l}\text { No hay jurisdicción penal } \\
\text { juvenil }\end{array}$ \\
\hline Suiza & $10 / 15^{* *}$ & $18 * * * * * *$ & $10-18$ \\
\hline
\end{tabular}

${ }^{6}$ También aquí se fija, por ejemplo para el caso de instrucciones en la ejecución de sanciones penales la imputabilidad a los 16 años, tanto que entre los 12 y 16 años pueden ser aplicados en todos los casos el encarcelamiento.

${ }^{7}$ No obstante la subida de la minoría de edad para comenzar la persecución penal de 8 a 12 años, generada por las críticas del Consejo de Europa, se mantienen las audiencias infantiles desde los ocho años como reacción del sistema tutelar. Véase BURMAN / JoHNSTONE / FrASER / MCNEILL (2011), pp. 1149 y ss. 
Dünkel: Edad de imputabilidad penal y jurisdicción de los tribunales juveniles en Europa

\begin{tabular}{|c|c|c|c|c|}
\hline \multicolumn{2}{|l|}{ Escocia } & $12^{* * * * * / 16}$ & $16 / 21$ & \begin{tabular}{|lll} 
8-16 (Audiencias infantiles); & (Tribunal penal \\
$16-18$ & (T) \\
juvenil) & &
\end{tabular} \\
\hline \multicolumn{2}{|l|}{ Serbia } & $14 / 16^{* *}$ & $18 / 21$ & $14-21$ \\
\hline \multicolumn{2}{|c|}{ Eslovaquia } & $14 / 15$ & $18 / 21$ & Tribunales penales generales \\
\hline \multicolumn{2}{|c|}{ Eslovenia } & $14 * * * * / 16$ & $18 / 21$ & $14-18$ \\
\hline \multicolumn{2}{|c|}{ España } & 14 & 18 & $14-18$ \\
\hline \multicolumn{2}{|c|}{ República Checa } & 15 & $18 / 18+$ & $15-18$ \\
\hline \multicolumn{2}{|c|}{ Turquía } & 12 & $15 / 18$ & $12-18$ \\
\hline \multicolumn{2}{|c|}{ Ucrania } & $14 * * * * / 16$ & $18 / 21$ & Tribunales penales generales \\
\hline \multicolumn{2}{|l|}{ Hungría } & $12^{* * * * / 14}$ & \begin{tabular}{|l|}
18 \\
\end{tabular} & $12-18$ \\
\hline \multicolumn{2}{|l|}{ Chipre } & 14 & $16 / 18 / 21$ & $14-18$ \\
\hline \multicolumn{5}{|c|}{$* \quad$ Solo las atenuantes del derecho penal general } \\
\hline \multicolumn{5}{|c|}{ ** Edad mínima para imponer sanciones - Ejecució } \\
\hline \multicolumn{5}{|c|}{ Solo para delitos de tráfico de estupefacientes } \\
\hline \multicolumn{5}{|c|}{ **** Solo para algunos delitos graves } \\
\hline \multirow{2}{*}{\multicolumn{5}{|c|}{$\begin{array}{l}* * * * * \text { Aplicación de medidas de protección propias del sistema tutelar, no hay responsabilidad penal i.e.S. } \\
* * * * * * \\
\text { El derecho penal de adultos suizo contempla como medida el internamiento especial }\end{array}$}} \\
\hline & \multicolumn{4}{|c|}{ ****** El derecho penal de adultos suizo contempla como medida el internamiento especial } \\
\hline
\end{tabular}

En relación con la edad de imputabilidad se considera en algunos países el concepto de la imputabilidad relativa (por ejemplo en Alemania parágrafo 3 de la Ley de Tribunales Juveniles, JGG) en el que los menores de 18 años tendrán responsabilidad penal solo cuando tengan capacidades cognoscitivas para entender el acto. Este concepto se encuentra en Italia, y desde 2002, en Estonia, desde el 2003 en República Checa y desde el 2005 para los adolescentes de 14 años en Eslovaquia. ${ }^{8}$ En Suiza el examen de responsabilidad es exigido si el tribunal quiere imponer sanciones penales en vez de las medidas educativas.

\subsection{Inclusión de los jóvenes adultos}

La discusión sobre los límites de aplicación del derecho penal juvenil no se agota con la discusión desde cuándo debe aplicarse, sino que también debe considerar la discusión del hasta cuándo deben ser aplicadas sus normas, es decir, debe hacerse cargo de la problemática de los menores adultos.

Existen dos modelos: por un lado el modelo alemán, regulado en el parágrafo 105 JGG, esto es, el derecho penal juvenil, con consideración al grado de madurez o, dicho de otra forma, se incorpora al joven adulto al derecho penal juvenil cuando la aplicación de la sanción penal juvenil aparece para él como la más idónea. Existe el modelo del joven adulto como circunstancia atenuante en el marco de aplicación del derecho penal general. La primera variante, la aplicación del derecho penal juvenil, es de acuerdo con el estudio de Greifswald, seguido en

${ }^{8}$ Véase PRUIN (2011), pp. 1566 y ss.; en Eslovaquia el concepto de discernimiento fue tomado del derecho francés y permitía aplicar el derecho penal de adultos entre los 14 y 15 años; también en Rumania. 
20 de 35 países. En Dinamarca, Alemania, Inglaterra y Gales, Finlandia, Italia, Portugal, Suecia y Suiza siguen los dos modelos, es decir, contemplan la aplicación del derecho penal juvenil como también atenuantes en el derecho penal general.

La tendencia actual, basada en la nueva evidencia levantada por la psicología del desarrollo y de la neurobiología, propone ampliar aún más los ámbitos de aplicación del derecho penal juvenil para los jóvenes adultos. En los hechos, como en la discusión alemana de la década del 50 y actualmente en Holanda, se discute la ampliación del derecho penal juvenil para los jóvenes infractores hasta los 24 años. ${ }^{10}$ Holanda, producto de los nuevos estudios de la neurociencia, ha decidido aplicar el derecho penal juvenil hasta la edad de 23 años. ${ }^{11}$

Las Reglas Europeas de 2008 (ERJOSSM), en consideración a estos nuevos estudios, han establecido en el principio 17: Los infractores jóvenes adultos ${ }^{12}$ podrán ser considerados, cuando proceda, como infractores menores de edad y se les tratará en consecuencia.

El principio Nr. 17 se inspira en las propuestas de la recomendación Nr. 11 de 2003, la que abogaba por la inclusión de los jóvenes adultos en el derecho penal juvenil. Las recomendaciones renuncian a los conceptos como los contenidos en el parágrafo 105 JGG (madurez progresiva) y abren con ello la posibilidad de aplicación del derecho penal juvenil o bien de algunas de las sanciones contempladas en su catálogo a los jóvenes adultos.

El comentario de las Reglas Europeas considera que los jóvenes adultos se encuentran en una etapa de transición producto de un alargamiento del proceso de formación y tardía madurez social, lo que haría necesario entregarles el estatus de adolescente e introducir en los sistemas de justicia alguno de los modelos, ya sea el de las atenuantes o bien el que derechamente los considera como adolescentes. Como vimos antes, la gran mayoría de los países han considerado esta etapa de transición y reglas de alguno e incluso de los dos modelos. Con todo, debemos decir que cada cierto tiempo aparecen voces que van en sentido contrario, voces anacrónicas, como en Alemania con los proyectos de los partidos CDU/CSU, los que han intentado limitar las normas del parágrafo 105 JGG y su aplicación práctica. ${ }^{13}$

${ }^{9}$ WeIJERS y GRISSO (2009), pp. 65 y ss.; JUNGER-TAS, y DÜNKEL (2009), p. 224; véase BONNIE / CHEMERS / SCHUCK (2012), especialmente el Capítulo 4 (Adolescent Development) y 5 (A Developmental Framework forReform); LOEBER / HOEVE / FARRINGTON / HOWELL / SLOT / VAN DER LAAN (2012), pp. 336 y ss.).

10 DVJJ (2002), p. 10 y s.; LOEBER / HOEVE / FARRINGTON / HOWELL / SLOT / VAN DER LAAN (2012), pp. 368 y ss. y pp. 394 y ss.

11 Loeber / HoEve / FARrington / Howell / Slot / VAN Der LAAN (2012); DÜNKEL y CASTRO (2012).

12 Según la definición legal de la regla 21.2 de las Reglas Europeas (ERJOSSM) se consideran adultos jóvenes los que se encuentran entre 18 hasta los 21 años, al igual que en el derecho penal juvenil alemán.

13 Véase PRUIN (2007); DÜNKEL y PRUIN (2011); DÜNKeL y PRUIN (2012). 


\section{Jurisdicción penal juvenil}

\subsection{Jueces especializados}

Desde el punto de vista histórico, el nacimiento del derecho penal juvenil viene de la mano con el nacimiento de los tribunales especiales que se establecen para los infractores menores de edad. ${ }^{14}$ Incluso las leyes penales juveniles llevan el nombre de los tribunales especiales, lo que da cuenta de que el movimiento de reforma del derecho penal juvenil ha estado desde sus inicios estrechamente vinculado con los tribunales juveniles.

Los jueces especializados con enfoque educativo han sido establecidos en la gran mayoría de los países europeos en las décadas del 20 y 30. Con la escisión de Europa, especialmente después de la Segunda Guerra Mundial, se debilita en los países de Europa del Centro y del Este el papel de los tribunales juveniles, debido a que se consideraba que el propio derecho penal -de corte socialista- podía con sus fines asegurar la función educativa.

En la década de los 90 se levanta nuevamente en este sector de Europa la discusión sobre la necesidad de reinstalar los tribunales juveniles. Fuera de Polonia, país de tradición tutelar, que en 1982 introduce los tribunales de familia, surgen los tribunales juveniles en Eslovenia en 1995, en la República Checa en 2003 y en Serbia en 2005. En Rusia y Rumania, no obstante el permanente rechazo del parlamento Ruso (Duma), se instaura en los tribunales ordinarios de las grandes ciudades una sala especializada. En Estonia, como en Lituania hay, dentro de los tribunales, comités juveniles especiales los que conocen delitos de baja gravedad y deciden la aplicación de medidas de diversión, siendo la sanción más aplicada la amonestación. ${ }^{15}$

Otra excepción se encuentra en los países escandinavos los que consideran grupos de decisión dentro de instituciones de bienestar que no pertenecen al Poder Judicial. En estas instituciones se considera con bastante fuerza la idea de la sanción como un bien para el adolescente. Como ejemplo, se puede mencionar Suecia donde el sistema traspasa la gran mayoría de los casos a las instituciones tutelares quedándoles a los tribunales muy poco espacio de decisión. ${ }^{16}$ Lo mismo se aplica a la reforma danesa de $2010 .^{17}$

En total, ocho de los treinta y cinco países no tienen ninguna clase de jurisdicción especial. Pertenecen a este grupo los países escandinavos, los Estados

\footnotetext{
14 Véase el análisis de 30 países europeos en GENSING (2014).

${ }^{15}$ GinTER y SOOTAK (2011), pp. 399 y ss.

${ }^{16}$ En Suecia el movimiento legislativo que pretendía instaurar tribunales juveniles especiales fue en 2001 rechazado. Véase GENSING (2011), p. 1616.

${ }^{17}$ GENSING(2014).
} 
Bálticos, Eslovaquia, Ucrania más Rusia y Rumania, los que solo tienen salas especializadas en las ciudades más pobladas.

En los demás países, como lo recomiendan las Reglas de Beijing y las Recomendaciones del Consejo de Europa, impera el modelo de la judicatura especial. Esta judicatura se contempla en primera y en segunda instancia, dejando la instancia de nulidad para las Cortes Supremas de justicia.

La organización de los tribunales presenta marcadas diferencias. En Escocia, bajo un fuerte tinte tutelar, se desarrolla en 1971 el sistema de audiencias infantiles, en las que se pueden conocer causas con niños imputados desde ocho años. ${ }^{18}$ En 2010 se introducen tribunales juveniles.

En Irlanda del Norte se sigue el modelo de Nueva Zelanda con las conferencias juveniles que se estructuran bajo la lógica de la reparación (justicia restaurativa). ${ }^{19}$ En Bélgica se instauran, con la reforma de 2007, las conferencias familiares, las que siguen también la lógica antes indicada. En Inglaterra y Gales hay nuevas orientaciones que giran en torno al modelo de conferencias escandinavo producto del crítico análisis de la política represiva de los últimos diez años. ${ }^{20}$

Una judicatura especial no significa el cumplimiento automático del principio de la especialidad. En Alemania las exigencias en torno a la especial experiencia y capacitación que deben tener los jueces y los fiscales juveniles (parágrafo 37 JGG) son en la práctica difíciles de cumplir. ${ }^{21}$ En la mayoría de los países las normativas exigen especiales conocimientos para los jueces y fiscales en similares términos que en Alemania. En otros países como Bulgaria, Chipre, Inglaterra y Gales $^{22}$ las legislaciones no contemplan ninguna exigencia para los jueces y fiscales.

\footnotetext{
${ }^{18}$ Burman / JOHNSTONE / Fraser / MCNEILl (2011), pp. 1150 y s. y pp. 1174 y ss.

19 O’MAHONY (2011), pp. 957 y ss.

${ }^{20}$ En el ámbito académico se venía hace tiempo criticando la política criminal. Títulos como "Time for a freshstart" de la Police Foundation o el de Smith 2010 "A New Response to Youth Crime" apelan por un vuelco en la política criminal. Véase GOLDSON (2011).

21 AdAm / Albrecht / PFeIfFer (1986); SimOn (2003); Drews (2005). El notorio déficit de las capacitaciones en temas de educación fue discutido en el proyecto de ley STORM-Gesetz de 2011, el que consideraba, como aspecto central, una efectiva y calificada consideración de los intereses de la víctima, la necesaria calificación de los fiscales y jueces juveniles en temas de pedagogía, pedagogía social, criminología y psicología juvenil y la necesaria capacitación de los operadores que comenzaban a trabajar en el sistema de justicia juvenil. Lamentablemente el proyecto fracasó por la oposición de algunos Estados Federales. Véase EISENBERG (2014)』37, pp. 443 y ss.

22 Incluso no es necesario que sean jueces profesionales.
} 


\section{2 Ámbito de competencia de las jurisdicciones penales juveniles}

\subsubsection{Principios}

Los jueces juveniles, en todas las legislaciones que prevén una judicatura especial, son competentes para conocer los casos de adolescentes infractores menores de 18 años. En los países que tienen un sistema de corte tutelar, los jueces resuelven no solo las infracciones penales, sino también casos civiles o cualquier tipo de conducta divergente que no necesariamente constituya delito, por ejemplo, en Polonia, Bélgica, Portugal, Escocia y en los Comités Juveniles de Bulgaria y Estonia.

Una excepción la constituyen países como Inglaterra y Gales, Irlanda del Norte y Escocia, los que han introducido las órdenes de comportamiento antisocial, que le otorgan a las infracciones juveniles un carácter civil. En todo caso, la desobediencia de órdenes es conocida por los tribunales juveniles.

En Bulgaria fallan los conocidos Comités Juveniles, que son competentes para conocer delitos y conductas antisociales como el ausentismo en las escuelas o la vagancia, pero con un limitado catálogo de sanciones, porque la prisión solo puede ser decidida por los tribunales de justicia. ${ }^{23}$

En otros países, la judicatura no es autónoma y forma parte del sistema general de tribunales. Sin embargo, es común en estos países que en primera y segunda instancia se contemple una sala especial para conocer los casos relativos a los jóvenes infractores.

En algunos de los países los tribunales juveniles son competentes para conocer relevantes infracciones penales cometidas por niños que están por debajo del umbral de la imputabilidad. Este es el caso de los tribunales juveniles de Francia que conocen de infracciones cometidas por niños desde los 10 años; en Grecia, desde los 8 años; en Portugal, entre los 12 y los 15 años y; en Escocia desde los ocho años. ${ }^{24}$

\subsubsection{La problemática de los jóvenes adultos}

Anteriormente comentamos -a propósito de la aplicación material del derecho penal juvenil- la problemática de los jóvenes adultos. En torno a los tribunales nace una pregunta equivalente referente a quién conocerá y decidirá los delitos ejecutados por este grupo. A diferencia de la problemática del derecho penal

${ }^{23}$ KANEV / FurTunOVA / ROUSSINOVA / BEKIRSKA(2011), p. 145.

${ }^{24}$ DÜNKEL / GRZYWA / PRUIN / ŠELIH (2011), pp. 1846 y ss. (Tabla 1). 
juvenil material, aquí el modelo predominante no es el alemán (Art. 108 JGG). Solamente Austria y Croacia siguen este modelo. ${ }^{25}$

En la gran mayoría de los países rige el principio de que la competencia está determinada por la edad al momento del hecho, de tal forma que, si al producirse la ejecución de la acción típica el sujeto activo es menor de 18 años, será juzgado por los tribunales juveniles, aunque la condena sea impuesta siendo este mayor de edad.

Otros países contemplan reglas especiales que se centran en el momento en que comienza el procedimiento judicial. Así, serán competentes los tribunales de los adultos si al momento del inicio del proceso el imputado tiene 19 años (República Checa), 21 (Serbia-Eslovenia), 23 (Croacia) o 25 años (Italia). En Bulgaria, en cambio, cesa la competencia de los tribunales juveniles si al momento de la dictación de la sentencia el acusado alcanza la mayoría de edad. ${ }^{26}$

\subsubsection{Los waiver: El resquebrajamiento del derecho penal juvenil}

Bajo la influencia norteamericana se inicia, en las décadas del 80 y del 90, una tendencia dirigida a disminuir las dimensiones de la jurisdicción penal juvenil. Bajo el título de "procedimientos de traslado" se propuso transferir hacia los tribunales de adultos los casos de criminalidad grave cometida por adolescentes menores de edad. Bajo esta tendencia no se delinearon criterios $\operatorname{claros}^{27}$ y en la actualidad se encuentran casos como el alemán que prevé el traslado a los 23 años y en el otro extremo casos como el de Inglaterra y Gales donde se permite el traslado a los 10 años. ${ }^{28}$

En Bélgica y Holanda la transferencia hacia los tribunales de adultos es posible entre los 16 y 17 años. En Bélgica fueron inicialmente los delitos de tránsito, como los de criminalidad leve objeto de transferencia, y en 2007 se incorporaron los delitos graves. En Holanda la aplicación de la transferencia ha ido disminuyendo en el tiempo, por ejemplo en 1995 el 16\% de los jóvenes infractores fueron trasladados a los tribunales de adultos y en el 2004 , solo $1,2 \%{ }^{29}$

En Irlanda, Irlanda del Norte y en Serbia pueden ser objeto de traslado los delitos terroristas y los delitos violentos. En Inglaterra y Gales es posible la

25 Aquí es decisivo la edad al momento en que se cometió el delito. En Croacia, sin embargo, se establecen limitaciones en el caso de los jóvenes adultos. En estos casos los tribunales de adultos son competentes si al momento de la condena el acusado tiene 23 años. Véase BOJANIC (2011), pp. 187 y ss. y GENSING (2014).

26 GENSING (2014).

27 Véase STUMP (2003); BishOP (2009), pp. 89 y ss.

${ }^{28}$ BisHOP (2009), pp. 89-90.

${ }^{29}$ Esto depende sin embargo del ámbito de aplicación de la sanción. El criterio hasta 1995 era seis meses, desde entonces dos años. Por lo tanto rara vez debían los jueces juveniles trasladar casos a los tribunales adultos para obtener una sanción más justa. Véase, PRUIN (2011), p. 1571. 
transferencia a la Corte de la Corona solo en pocos casos (vinculados con delitos de extrema violencia).

El aumento de las transferencias no siempre ha estado ligado con el endurecimiento de las sanciones. Por ejemplo en Francia se permitió el traslado para hacer posible la aplicación de multas en procedimientos sumarios. ${ }^{30}$ En Alemania esta estrategia también ha sido seguida, por ejemplo, en el caso de delitos del tránsito cometidos por jóvenes adultos. ${ }^{31}$

\subsection{Organización de tribunales juveniles y participantes en el proceso}

\subsubsection{Jueces juveniles}

En la gran mayoría de los países europeos existen tribunales juveniles, por ejemplo, en Austria, Francia, Alemania, Grecia, Hungría, Italia, Holanda, Polonia Serbia, Eslovenia, Turquía y España. Los sistemas procesales de estos países se caracterizan por una estructura organizativa de dos instancias, una competencia amplia que les permite conocer delitos graves y fallar con lógicas educativas.

Una particularidad se encuentra en los países del Este y Centro de Europa con la figura de los comités juveniles. Bajo la influencia socialista, a estos comités se les otorgaron funciones generalmente entregadas a los servicios encargados de la protección de menores, es decir, no solo tienen facultades para sancionar los delitos de bagatela, sino además conductas desviadas como ausentismo escolar, vagancia, abandono del hogar, etc.

Los comités juveniles son interdisciplinarios y tienen un corte pedagógico, incorporando no solo a docentes, trabajadores sociales y psicólogos, sino además a ciudadanos comprometidos. La función de esta institución puede ser comparada, en algunas dimensiones, con las instituciones de protección de la infancia que colaboran con la justicia. Si bien estos comités no tienen el estatus de un tribunal, están facultados, en los casos de criminalidad leve, para aplicar medidas procesales alternativas e imponer sanciones no privativas de libertad como amonestaciones y órdenes. Es por este motivo que se dice que estos Comités tienen una naturaleza híbrida porque comparten funciones propias de tribunales y de servicio de protección de menores.

30 PRUIN (2011), p. 1569.

31 PRUIN (2007); DÜNKEL / GRZYWA / HORSFIELD / Pruin (2011), pp. 589 y ss. 
Tabla 2: Jurisdicción de los Tribunales Juveniles: modelos de tribunales en el derecho penal juvenil en Europa

\begin{tabular}{|c|c|}
\hline Juzgado Juvenil o Tribunal juvenil, u otra denominación & Tribunales penales generales \\
\hline $\begin{array}{l}\text { Bélgica (Conferencias familiares) } \\
\text { Bulgaria (Cámara con una sala especial; y también actúan los Comités } \\
\text { juveniles) } \\
\text { Alemania (Tribunales juveniles) } \\
\text { Inglaterra/Gales (Magistrados pero sin especialización) } \\
\text { Francia (Tribunales juveniles) } \\
\text { Grecia (Tribunales juveniles) } \\
\text { Irlanda (Children's Court, pero juez no especializado) } \\
\text { Italia (Tribunal juvenil) } \\
\text { Croacia (Cámara especial) } \\
\text { Holanda (Tribunal juvenil) } \\
\text { Irlanda del Norte (Tribunal juvenil, pero las Conferencias juveniles } \\
\text { tienen prioridad) } \\
\text { Austria (Tribunales juveniles) } \\
\text { Polonia (Tribunales de la familia) } \\
\text { Portugal (Tribunales de la infancia y familia) } \\
\text { Rumania (Cámaras especiales tratan materias de familia y juventud) } \\
\text { Escocia (Children's Hearings System; pero también los Tribunales } \\
\text { juveniles en los casos de los 16 y } 17 \text { años; como también los } \\
\text { tribunales penales de adultos) } \\
\text { Suiza (Tribunales juveniles) } \\
\text { Serbia (Tribunales y Cámaras juveniles) } \\
\text { Eslovenia (Tribunales y Cámaras juveniles) } \\
\text { España (Tribunales juveniles) } \\
\text { República Checa (Cámaras especiales) } \\
\text { Hungría (Tribunales y Cámaras juveniles) }\end{array}$ & $\begin{array}{l}\text { Dinamarca } \\
\text { Finlandia } \\
\text { Suecia } \\
\text { Estonia (en la aplicación de la } \\
\text { Diversion son los Comités } \\
\text { Juveniles importantes) } \\
\text { Letonia } \\
\text { Lituania } \\
\text { Rusia (proyecto de tribunal } \\
\text { juvenil en Rostov, } \\
\text { Krasnoyarsk etc.; Comités } \\
\text { juveniles para Jóvenes } \\
\text { menores de } 16 \text { años) } \\
\text { Eslovaquia (Proyecto de } \\
\text { ley) }\end{array}$ \\
\hline
\end{tabular}

Fuente: GENSING (2014), pp. 300 y ss.

En lo que se refiere a las causales de impugnación, los adolescentes se encuentran en una situación desmejorada a la de los adultos. Así por ejemplo en Alemania el parágrafo 55 Abs.2 JGG da la posibilidad de opción entre apelación y nulidad, ejercicio que hace inmediatamente inadmisible en cada caso el otro recurso. ${ }^{32}$ También en Grecia después de la reforma de corte alemán aprobada en el 2010 se restringió la interposición de recursos judiciales. ${ }^{33}$ Con esta reducción de los recursos procesales se vulneran los estándares internacionales del debido proceso, en concreto el artículo 40 II b) v) de la Convención de los Derechos del Niño, en la medida en que se limita el ámbito de decisiones que pueden ser sometidas a una autoridad judicial superior. También se vulneran las Reglas de Beijing Nr.7.1 y las Reglas Europeas Nr.13 (ERJOSSM), puesto que estas restricciones disminuyen los

\footnotetext{
${ }^{32}$ Hay que atender que los países también dan a los adultos una doble instancia. Así por ejemplo en Suiza, véase. HEBEISEN (2011), pp. 1405 y ss.; véase también GENSING (2014), cap. 6.6.2.

33 VÁRADI-CSEMA (2011), pp. 671 y ss. y p. 686.
} 
derechos y garantías de los jóvenes en comparación con los reconocidos a los adultos en el proceso penal. ${ }^{34}$

\subsubsection{Participantes en el proceso penal}

Generalmente participan en el proceso juvenil el imputado, el fiscal, el juez, el tutor, el defensor, la víctima y los órganos asesores, como por ejemplo, los servicios sociales encargados de la niñez e infancia.

En el caso del imputado se plantea la pregunta en torno a la comparecencia obligatoria y con ello las situaciones especiales en que puede ser excluido. La discusión gira también en torno al alcance de su derecho de participación en el juicio. En Italia por ejemplo, se puede renunciar al derecho de interrogar, ${ }^{35}$ cuestión que en Inglaterra y Gales no está permitida porque significaría una vulneración de la Convención de los Derechos del Niño, específicamente en su artículo 402 b iv. En todo caso, en casi todos los países analizados la participación activa del adolescente en su defensa tiene un reconocimiento general.

En Europa los fiscales especializados, a diferencia de los jueces, son escasos. Esto se debe al especial protagonismo que tienen los jueces en la etapa de investigación ${ }^{36} \mathrm{o}$ al dominante rol de la policía. En torno a las tareas que competen a los fiscales, en algunos estados alemanes no solo persiguen, sino que se encargan de la ejecución de la sanción (también en Serbia y en Holanda).

En Alemania, la participación del tutor ha sido muy discutida como consecuencia de un fallo del Tribunal Constitucional Federal Alemán (parágrafo 51, 2 JGG). Básicamente el derecho de los tutores a estar presentes en el procedimiento ha sido relativizado permitiendo la normativa que estos sean excluidos como testigos. ${ }^{37}$

En derecho comparado la participación del tutor tiene una variada amplitud, la que se encuentra vinculada con las etapas en que él puede participar (etapa de investigación o juicio oral), y con el ejercicio de determinados derechos en el proceso (interrogar, presentar pruebas, presentar recursos). Resulta interesante que en varios países la situación de quedar al cuidado de los padres (tutores), importa una medida que evita la prisión preventiva.

Una tendencia de los últimos años es la imputación de los padres de los jóvenes que cometieron delitos. Bajo el marco de las órdenes parentales, podrían

\footnotetext{
34 DÜNKEL (2011), p. 144.

35 GENSING (2013), cap. 5.1.2.

36 Así por ejemplo los jueces juveniles en Francia, Croacia, Serbia, Eslovenia y en algunos cantones en Suiza. Véase GENSING (2013), cap. 4.2.2.

${ }^{37}$ BVerfG DVJJ-Journal 2003, pp. 68 y ss; la nueva regulación del $\int 51$ Abs. 2 JGG fue incluida en la Ley de Modernización de la Justicia del 22.12.2006.
} 
ser condenados desde al pago de una multa hasta la participación obligatoria en un curso de educación de hijos. Detrás de esta sanción se encuentra el reproche que se le hace al padre por no preocuparse suficientemente por la educación del hijo que delinque. Esta tendencia de condenar a los padres desmotivados a pagar multas o participar en cursos, no obstante las críticas por su falta de eficacia, ha sido implementada en Inglaterra y Gales, Bulgaria, Irlanda y Escocia. Los cursos para los padres podrían tener mayor sentido bajo proyectos de prevención del delito y centrarse en padres motivados que quieran sacar a sus hijos de la delincuencia. ${ }^{38}$

Por su parte, la intervención de las instituciones sociales en el proceso se enmarca dentro del carácter educativo que se le otorga al derecho penal juvenil. En Alemania se les da a estas instituciones un rol central que se evidencia no solo en la precisa regulación de los parágrafos 38, 72a y 72b JGG, sino además en el carácter obligatorio de la intervención de estas instituciones en el proceso. En Europa hay una variedad de pedagogos sociales, psicólogos y otras profesiones de las ciencias sociales que colaboran en el proceso desde la etapa de investigación hasta la determinación de la sanción. Un ejemplo de equipos interdisciplinarios puede encontrarse en Inglaterra y Gales con los "equipos de jóvenes infractores". En general estos servicios pueden depender derechamente de una institución social, del Ministerio de Justicia, los tribunales e incluso del Ministerio Público.

Tabla 3: $\quad$ Instituciones sociales que intervienen en el proceso juvenil

\begin{tabular}{|l|l|}
\hline País & Institución y su dependencia \\
\hline Bélgica & Servicio social (depende del Tribunal) \\
\hline Bulgaria & $\begin{array}{l}\text { No hay una institución especial; pero en la interrogación se contempla la } \\
\text { participación de un educador o pedagogo }\end{array}$ \\
\hline Dinamarca & Servicio social \\
\hline Alemania & Servicio colaborador de los Tribunales juveniles (Jugendamt) \\
\hline Inglaterra/Gales & Grupo interdisciplinario "Youth Offending Teams" \\
\hline Estonia & No hay institución especial \\
\hline Finlandia & $\begin{array}{l}\text { Delegados encargados de libertad condicional que trabajan en estrecha } \\
\text { relación con instituciones tutelares }\end{array}$ \\
\hline Francia & $\begin{array}{l}\text { Servicio colaborador de los Tribunales juveniles (Service éducatif auprés des } \\
\text { tribunaux) }\end{array}$ \\
\hline Grecia & Servicio colaborador de los Tribunales juveniles \\
\hline Irlanda & $\begin{array}{l}\text { Delegados encargados de libertad condicional que trabajan en estrecha } \\
\text { relación con instituciones tutelares }\end{array}$ \\
\hline Italia & Servicio social (Dependiente del Ministerio de Justicia) \\
\hline Croacia & $\begin{array}{l}\text { Institución tutelar; pero también pertenecen al tribunal profesionales } \\
\text { capacitados como trabajadores sociales o psicólogos. }\end{array}$ \\
\hline Letonia & $\begin{array}{l}\text { No hay una institución especial; pero se contempla en la determinación de la } \\
\text { sanción la intervención de un especialista, educador o psicólogo }\end{array}$ \\
\hline
\end{tabular}

${ }^{38}$ KRÜGER (2010); LÖSEL (2012). 


\begin{tabular}{|l|l|}
\hline País & Institución y su dependencia \\
\hline Lituania & $\begin{array}{l}\text { No hay una institución especial; pero se contempla en la interrogación la } \\
\text { participación de un representante del servicio de protección al menor o un } \\
\text { psicólogo }\end{array}$ \\
\hline Holanda & Servicio de protección al menor \\
\hline Irlanda del Norte & "Social Service Department" \\
\hline Austria & $\begin{array}{l}\text { Servicio colaborador de los Tribunales juveniles (excepcionalmente delegados } \\
\text { de libertad condicional) }\end{array}$ \\
\hline Polonia & Centro de diagnóstico y asesoría familiar \\
\hline Portugal & Servicio Social (Ministerio de Justicia) \\
\hline Rumania & Delegados de la libertad condicional \\
\hline Rusia & $\begin{array}{l}\text { No hay autoridades especiales, pero en el interrogatorio participan pedagogos } \\
\text { o psicólogos }\end{array}$ \\
\hline Escocia & Trabajadores sociales (Instituciones comunales) \\
\hline Suecia & Instituciones sociales \\
\hline Suiza & Servicio social dependiente del tribunal \\
\hline Serbia & $\begin{array}{l}\text { Instituciones de tutela, pero también profesionales especializados dependientes } \\
\text { del tribunal: pedagogos y psicólogos }\end{array}$ \\
\hline Eslovaquia & Instituciones tutelares \\
\hline Eslovenia & Centro para el trabajo social \\
\hline España & Equipos técnicos \\
\hline República Checa & Instituciones tutelares \\
\hline Hungría & Delegado de libertad condicional \\
\hline Fuente: & (2014), cap 5.4 toba 6 \\
\hline
\end{tabular}

Fuente: GENSING (2014), cap. 5.4, tabla 6.

En lo referente al rol de los defensores hay en Europa interesantes perspectivas que pueden ser analizadas. La tradicional defensa obligatoria en los países del Centro y del Este de Europa fue ampliada y es similar a los países del Oeste. ${ }^{39}$ Sin embargo la pregunta que debemos hacernos es si después de la caída del muro, el derecho a un defensor legal ha sido especialmente consagrado en las leyes juveniles. En algunos países del Este como Alemania (parágrafo 68 JGG), Francia, Suecia y Austria hay un especial reconocimiento del derecho a defensa para el caso de los adolescentes. Pero la regla general va en el otro sentido y es difícil encontrar en las legislaciones un reconocimiento expreso a tal derecho, por ejemplo, en Inglaterra y Gales, Dinamarca, Finlandia y Grecia.

La participación de la víctima no solo ha sido objeto de reformas en Alemania. La introducción de la acción adhesiva, la reparación del mal causado y la mediación son formas en que el rol de la víctima ha sido reconocido en el proceso.

La fuerte predominancia de los intereses de las víctimas es un fenómeno global que se manifiesta en el general y particular fortalecimiento de los derechos del lesionado y el mejoramiento de su posición en el proceso, predominancia que en Alemania ha sido concretizada con las reformas a la Ley del Derecho de las Víctimas de 1986 y 2004 (OpferschutzG) y que ha tenido sus pares en todos los países europeos. Solo en raras ocasiones se encuentran limitaciones a los derechos

${ }^{39}$ GENSING (2013), cap. 5.5 . 
procesales de las víctimas en pos de salvaguardar los intereses del imputado o por motivos de reinserción. Así ocurre, por ejemplo, en Italia, Croacia, Eslovenia y Hungría. $^{40}$

En lo que se refiere a las particularidades del derecho procesal penal -con excepción de Inglaterra y Eslovenia- la acción privada es inadmisible.

La adhesión al proceso, esto es la presentación de una persona privada que dispone de derechos propios para actuar en el proceso, se ha ampliado y solo en pocos países como en Alemania, Italia, Polonia, Portugal, Inglaterra y Gales se excluye. Pero debe considerarse que en estos países las pretensiones de la víctima para la compensación o la reparación son escuchadas. ${ }^{41}$

La acción de adhesión tampoco está permitida para los jóvenes imputados en los países escandinavos. Con todo, debería ser más adecuado en el contexto de un derecho procesal penal juvenil educativo la aplicación de medidas restaurativas más que el fortalecimiento del acusador adhesivo. En los hechos, las reformas penales de los últimos tiempos le han otorgado a la mediación o los grupos de conferencia un importante protagonismo. ${ }^{42}$

\section{Perspectivas}

El derecho penal juvenil del futuro debería ampliar su ámbito de aplicación e incorporar a los jóvenes adultos. Los nuevos descubrimientos de la psicología del desarrollo y de la neurociencia obligan a replantear la edad de ingreso de los jóvenes adultos removiendo la barrera de los 18 años. ${ }^{43}$ En los hechos, la evidencia científica identifica los 25 años como la edad en que se logra la madurez de las estructuras de decisión y responsabilidad en una persona. Esta evidencia ha jugado un importante rol en recientes reformas, como la emprendida en Holanda en 2014 donde el derecho penal juvenil es aplicable hasta los 23 años y ha venido a ratificar las legislaciones de Alemania, Austria y Croacia. ${ }^{44}$ También en Estados Unidos ha tenido acogida con el Informe del Consejo Nacional de Investigación de la Academia Nacional, el que recomienda reforzar los viejos paradigmas del debido proceso y la orientación educativa y así disminuir la transferencia de los jóvenes al derecho penal de los adultos. ${ }^{45}$ En la medida en que las reformas penales juveniles recojan esta evidencia y se concentren en ampliar hacia arriba las fronteras del

40 Ibid, cap. 5.6.

${ }^{41}$ Ibid, cap. 5.6.2.

42 DoAky O’Mahony (2011), pp. 1717 y ss.; DünKel / GrZYWa / Pruin / SEliH (2011).

43 Acuerdos del Deutschen Juristentags NJW (2002), pp. 3077 y ss.; véase también la pretensión de tener un derecho para los jóvenes infractores hasta los 24 años en STRENG (2012), p. 50 y s.

44 Bishop (2009), p. 101.; LOEBER / HOEVE / FARRINGTON / HOWELL / VAN DER LAAN (2012), pp. 368 y ss. y pp. 394 y ss.

45 STUMP (2003); BISHOP (2009). 
Dünkel: Edad de imputabilidad penal y jurisdicción de los tribunales juveniles en Europa

derecho penal juvenil enfrentaremos una nueva época de cambios y desafíos que deberían materializarse en los próximos años y que configurarán el futuro del derecho penal y procesal penal de los adolescentes. 


\section{BIBLIOGRAFÍA}

* Adam, Hildegard/Albrecht, Hans-Jörg/PFeIfFer, Christian (1986): Jugendrichterund Jugendstaatsanwälte in der Bundesrepublik Deutschland (Freiburg, Max-Planck-Institut fur Auslandisches und Internationales Strafrecht).

* Bishop, Donna (2009): “Juvenile Transfer in the United States", en: Junger-Tas, J. y Dünkel, F. (Eds.) Reforming Juvenile Justice (Dordrecht, Heidelberg, London, New York, Springer).

* Bochmann, Christian (2009): Entwicklungeineseuropäischen Jugendstrafrechts (Baden-Baden, Nomos).

* Bojanic, Igor (2011): "Juvenile Justice Systems in Croatia", en: Dünkel, F./Grzywa, J./Horsfield, P./Pruin, I. (Eds.), Juvenile Justice Systems in Europe - Current Situation and Reform Developments, segunda edición (Mönchengladbach, Forum Verlag Godesberg), pp. 187-222.

* BONNIE, Richard/CHEMERS, Betty/SCHUCK, Julie (Eds.) (2012): Reforming Juvenile Justice: A Developmental Approach (National Research Council of the National Academies).

* Burman, Michele/Johnstone, Jenny/Fraser, Alistair/McNeill, Fergus (2011): "Juvenile Justice Systems in Scotland" en: Dünkel, F./Grzywa, J./Horsfield, P./Pruin, I. (Eds.), Juvenile Justice Systems in Europe - Current Situation and Reform Developments, segunda edición (Mönchengladbach, Forum Verlag Godesberg), pp. 1149-1196.

* Council of Europe (Ed.), (2009): European Rules for juvenile offenders subject to sanctions or measures- Recommendation (2008) 11 (Strasbourg: Council of Europe Publishing).

* DoAK, Jonathan y O’MAhony, David (2011): "Developing mediation and restorative justice for young offenders across Europe”, en: Dünkel, F./Grzywa, J./Horsfield, P./Pruin, I. (Eds.), Juvenile Justice Systems in Europe - Current Situation and Reform Developments, segunda edición (Mönchengladbach, Forum Verlag Godesberg), pp. 17171746.

* DoEK, Jaap (2009): “The UN Convention of the Rights of the Child”, en: Junger-Tas, J. y Dünkel, F. (Eds.), Reforming Juvenile Justice (Dordrecht, Heidelberg, London, New York, Springer), pp. 19-31.

* Drews, N. (2005): Die Aus und Fortbildungssituation von Jugendrichtern und Jugendstaatsanwälten in der Bundesrepublik. Deutschland - Anspruchund Wirklichkeit von \37 JGG (Aachen, Shaker Verlag).

* DüNKEL, Frieder (2008): "Jugend strafrecht im europäischen Vergleichim Lichtaktueller Empfehlungen des Europarats" en Neue Kriminal politik (20), pp. 102-114.

(2011): “Die Europäischen Grundsätze für die von Sanktionen oder Maßnahmen betroffenen jugendlichen Straftäter und Straftäterinnen ("European Rules for Juvenile Offenders Subject to Sanctions or Measures" ERJOSSM)" en Zeitschrift für Jugendkriminalrecht und Jugendhilfe (22), pp. 140-154.

* Dünkel, Frieder / GrZyWA, Joana / Horsfield, Philip / Pruin, Ineke (Eds.) (2011): Juvenile Justice Systems in Europe - Current Situation and Reform Developments, segunda edición (Mönchengladbach, Forum Verlag Godesberg).

* Dünkel, Frieder / GrZYWA, Joana / Pruin, Ineke / ŠElih, Alenka (2011): "Juvenile Justice in Europe - Legal aspects, policy trends and perspectives in the light of human rights standards”, en: Dünkel, F./Grzywa, J./Horsfield, P./Pruin, I. (Eds.), Juvenile Justice Systems in Europe - Current Situation and Reform Developments, segunda edición (Mönchengladbach, Forum Verlag Godesberg), pp. 1.839-1.898.

* Dünkel, Frieder / CASTRo, Alvaro (2012): "Reglas europeas para infractores menores de edad sometidos a sanciones o medidas", en Revista digital Maestría en Ciencias Penales (Nr. 4), (Costa Rica), pp. 93-122. 
Dünkel: Edad de imputabilidad penal y jurisdicción de los tribunales juveniles en Europa

* DüNKEL, Frieder / PRUIN, Ineke (2011): "Young adult offenders in the criminal justice systems of European countries”, en: Dünkel, F./Grzywa, J./Horsfield, P./Pruin, I. (Eds.), Juvenile Justice Systems in Europe - Current Situation and Reform Developments, segunda edición (Mönchengladbach, Forum Verlag Godesberg), pp. 1583-1606.

(2012): "Young adult offenders in the criminal justice systems of European countries”, en: Lösel, F./Bottoms, A./Farrington, D. P. (Eds.): Young Adult Offenders. Lost in transition? (Devon, Willan Publishing), pp. 11-38.

* DVJJ (2002): Vorschläge für eine Reform des Jugendstrafrechts, 2. JugendstrafrechtsreformKommission, en DVJJ-Journal-Extra (Nr. 5).

* Eisenberg, Ulrich (2014): Jugendgerichtsgeset\%. Kommentar, 17. Aufl. (München, C.H.Beck).

* Gensing, Andrea (2011): "Jurisdiction and characteristics of juvenile criminal procedure in Europe", en: Dünkel, F./Grzywa, J./Horsfield, P./Pruin, I. (Eds.), Juvenile Justice Systems in Europe - Current Situation and Reform Developments, segunda edición (Mönchengladbach, Forum Verlag Godesberg), pp. 1607-1648.

(2014): Jugendgerichtsbarkeit und Jugendstrafverfahren im europäischen Vergleich, (Mönchengladbach, Forum Verlag Godesberg).

* Ginter, Jaan / SoOTAK, Jaan (2011): "Juvenile Justice Systems in Estonia”, en: Dünkel, F./Grzywa, J./Horsfield, P./Pruin, I. (Eds.), Juvenile Justice Systems in Europe - Current Situation and Reform Developments, segunda edición (Mönchengladbach, Forum Verlag Godesberg), pp. 399-422.

* Goldson, Barry (2011): 'Time for a Fresh Start', but is this it? A Critical Assessment of The Report of the Independent Commission on Youth Crime and Antisocial Behaviour" en Youth Justice (11), pp. 3-27.

* Hebeisen, Dieter (2011): "Juvenile Justice Systems in Switzerland", en: Dünkel, F./Grzywa, J./Horsfield, P./Pruin, I. (Eds.), Juvenile Justice Systems in Europe - Current Situation and Reform Developments, segunda edición (Mönchengladbach, Forum Verlag Godesberg), pp. 1389-1440.

* HöynCK, Theresia / Neubacher, Frank / SCHÜler-Springorum, Horst (2001): Internationale Menschenrechtsstandard sund das Jugendkriminalrecht. Dokumente der Vereinten Nationen und des Europarats (Mönchengladbach, Forum Verlag Godesberg).

* Junger-Tas, Josie/Dünkel, Frieder (2009): "Reforming Juvenile Justice: European Perspectives", en: Junger-Tas, J. y Dünkel, F. (Eds.) Reforming Criminal Justice (Berlín, New York, Springer).

* Kanev, Krassimir / Furtunova, Daniela / Roussinova, Polina / BeKIRSKa, Yordanka (2011): "Juvenile Justice Systems in Bulgaria", en: Dünkel, F./Grzywa, J./Horsfield, P./Pruin, I. (Eds.), Juvenile Justice Systems in Europe - Current Situation and Reform Developments, segunda edición (Mönchengladbach, Forum Verlag Godesberg), pp. 131-186.

* KrÜGER, Maik (2010): Frühprävention dissozialen Verbaltens. Entwicklungen in der Kinder- und Jugendhilfe (Mönchengladbach, Forum Verlag Godesberg).

* KüHnE, Hans Heiner (1978): Strafverfahrensrecht als Kommunikationsproblem. Prolegomena einer strafverfahrensrechtlichen Kommunikationstheorie (Heidelberg, Kriminalistik Verlag).

(1996): "Parties to the proceedings in Germany, France and Spain", en: Cavadino, P. (Ed.) (2010): Children who kill (Winchester, WatersidePress), pp. 126-130.

(2010): Strafprozessrecht. Ein Lehrbuch zum deutschen und europäischen Strafverfahrensrecht, 8. Aufl. (Heidelberg, CF Müller Verlag).

* KüHne, Hans Heiner / Wyngaert, Christine/McAuley, Finbarr (Eds.) (1993): Criminal Procedure Systems in the European Community (Londres, Butter Worths).

* Loeber, Rolf / Hoeve, Machteld / Farrington, David / Howell, James / Slot, Wim/ VAN DeR LAAN, Peter (2012): “Overview, Conclusions, and Policy and Research Recommendations", en: Loeber, R./Hoeve, M./Slot, W./van der Laan, P. H. (Eds.): 
Persisters and Desisters in CrimefromAdolescenceintoAdulthood. Explanation, Prevention and Punishment. Farnham Surrey: Ashgate(Londres, Ashgate Publishing), pp. 335-412.

* LÖSEL, Friedrich (2012): “Offender treatment and rehabilitation: Whatworks?” en: Maguire, M., Morgan, R. y Reiner, R. (Eds.), The Oxford Handbook of Criminology, 5. Aufl. (Oxford University press), pp. 986-1016.

* Miyazawa, Koichi (1975): "Das japanische Jugendschutzgesetz mit Nebengesetzen. Eine kommentierte Übersetzung mit Einführung”, en: Sammlung Deutscher Strafgesetzbücher (Berlín, Gruyter Verlag).

* O’Mahony, David (2011): “Juvenile Justice Systems in Northern Ireland” en: Dünkel, F./Grzywa, J./Horsfield, P./Pruin, I. (Eds.), Juvenile Justice Systems in Europe - Current Situation and Reform Developments, segunda edición (Mönchengladbach, Forum Verlag Godesberg), pp. 957-990.

* Pruin, Ineke Regina (2007): Die Heranwachsenden regelung im deutschen Strafrecht. Jugendkriminologische, entwicklungspsychologische, jugendsoziologische und rechtsvergleichende Aspekte (Mönchengladbach, Forum Verlag Godesberg).

(2011): “The scope of juvenile justice systems in Europe”, en: Dünkel, F./Grzywa, J./Horsfield, P./Pruin, I. (Eds.), Juvenile Justice Systems in Europe - Current Situation and Reform Developments, segunda edición (Mönchengladbach, Forum Verlag Godesberg), pp. 1.539-1.582.

* Rodrigues, Anabela / Duarte-Fonseca, Antonio (2011): "Juvenile Justice Systems in Portugal”, en Dünkel, F./Grzywa, J./Horsfield, P./Pruin, I. (Eds.), Juvenile Justice Systems in Europe - Current Situation and Reform Developments, segunda edición (Mönchengladbach, Forum Verlag Godesberg), pp. 1027-1076.

* SIMOn, Kirsten (2003): Der Jugendrichterim Zentrum der Jugendgerichtsbarkeit - Ein Beitragzu Möglichkeitenund Grenzen des jugendrichterlichen Erziebungsauftrages in Hinblickauf $\int 37 J G G$ (Mönchengladbach, Forum Verlag Godesberg).

* Smith, David (Hrsg.) (2010): A New Response to Youth Crime (Cullompton: Willan Publishing).

* Stańdo-Kawecka, Bárbara (2011): "Juvenile Justice Systems in Poland”, en Dünkel, F./Grzywa, J./Horsfield, P./Pruin, I. (Eds.), Juvenile Justice Systems in Europe - Current Situation and Reform Developments, segunda edición (Mönchengladbach, Forum Verlag Godesberg), pp. 991-1026.

* Streng, Franz (2012): Jugendstrafrecht, 3. Aufl. (Heidelberg,C.F. Müller).

* Stump, Brigitte (2003): Adult time for adult crime - Jugendlichezwischen Jugend- und Erwachsenenstrafrecht (Mönchengladbach, Forum Verlag Godesberg).

* VÁradi-Csema, Erika (2011): "Juvenile Justice Systems in Hungary", en Dünkel, F./Grzywa, J./Horsfield, P./Pruin, I. (Eds.), Juvenile Justice Systems in Europe - Current Situation and Reform Developments, segunda edición (Mönchengladbach, Forum Verlag Godesberg), pp. 671-720.

* Weijers, Ido/Grisso, Thomas (2009): “Criminal Responsibility of Adolescents: Youth as Junior Citizenship”, en: Junger-Tas, J. y Dünkel, F. (Eds.) Reforming Criminal Justice (Berlín, New York, Springer), pp. 47-69. 